vor der Europawahl im Mai 2014 ein Ergebnis zu präsentieren und das Vertrauen in die Handlungsfähigkeit der Politik und den digitalen Markt in Europa zurückzugewinnen."

\section{Forderungspapier des BfDI und des Vorstands des Verbraucherzentrale Bundesverband (vzbv) an die neue Bundesregierung}

Zum Weltinternettag am 29.10.2013 forderten der Bundesbeauftragte für den Datenschutz und die Informationsfreiheit, Peter Schaar, und der Vorstand des Verbraucherzentrale Bundesverband (vzbv), Gerd Billen, im Rahmen einer gemeinsamen Bundespressekonferenz die neue Bundesregierung sowie die Abgeordneten des 18. Deutschen Bundestags auf, den Schutz personenbezogener Daten effektiv zu stärken.

\section{Wortlaut des Forderungspapiers}

Die Datenspuren, die Bürgerinnen und Bürger sowie Verbraucherinnen und Verbraucher nahezu bei jeder Aktion und Kommunikation - insbesondere im Internet - hinterlassen, sind nicht nur ein begehrter Rohstoff für Unternehmen, sondern auch eine verlockende Erkenntnisquelle für Sicherheitsbehörden. Verbraucher verlieren zunehmend die Kontrolle über ihre Daten. Von der neuen Bundesregierung erwarten wir, dass sie der Gefahr des „gläsernen" Bürgers und Verbrauchers energisch auf europäischer, internationaler und nationaler Ebene begegnet.

Konkret bedeutet das:

1. Zügige Verabschiedung der europäischen Datenschutzreform

Die Bundesregierung muss sich jetzt auf europäischer Ebene für eine zügige Verabschiedung EU-weit geltender Datenschutzstandards mit hohem Schutzniveau einsetzen. Dies umfasst unter anderem:

- hohe Transparenzvorgaben sowie verständliche und leicht zugängliche Informationen über Art und Umfang der Datenverarbeitung in prägnanter Form

- Stärkung der Betroffenenrechte unter Berücksichtigung der Langlebigkeit und Verfügbarkeit digitaler Daten, insbesondere der Rechte auf Datenlöschung und Datenübertragbarkeit

- Stärkung bestehender Verbraucher- und Datenschutzinstitutionen

2. Keine umfassende und anlasslose Überwachung der Verbraucherkommunikation durch Sicherheitsbehörden aus Drittstaaten

- Die Bundesregierung muss sich auf europäischer und internationaler Ebene dafür einsetzen, dass keine umfassende und an lasslose Überwachung der Verbraucherkommunikation erfolgt.

- Die Bundesregierung muss sich auf europäischer Ebene für eine kritische Bestandsaufnahme der Rechtsgrundlagen für die Übermittlung von Verbraucherdaten an Drittstaaten, wie das Safe-Habor-Abkommen oder das SWIFT-Abkommen und das PNR-Abkommen, einsetzen.

\section{Modernisierung des deutschen Datenschutzrechts}

Die Debatte zur Verbesserung des europäischen und internationalen Datenschutzes darf nicht davon ablenken, dass auch der Re- formstau beim deutschen Datenschutzrecht aufgelöst werden muss. Dies umfasst unter anderem:

- die Stärkung des Datenschutzes im Sozial- und Gesundheitswesen sowie einen verbesserten Beschäftigtendatenschutz

- die Einschränkung der Zugriffsmöglichkeit der Sicherheitsbehörden auf Verbraucherdaten, insbesondere im Rahmen der Vorratsdatenspeicherung

\section{BVDW: Kritik am Votum des LIBE-Ausschusses zur neuen EU Datenschutz-Grundverordnung}

Der Bundesverband Digitale Wirtschaft (BVDW) e.V., die zentrale Interessenvertretung der deutschen Digitalindustrie, hat am 22.10 2013 das am Abend zuvor vom federführenden Ausschuss für Bürgerliche Freiheiten, Justiz und Inneres (LIBE) getroffene Votum zur neuen EU Datenschutz-Grundverordnung für Europa als nicht ausreichend kritisiert.

Der verabschiedete Kompromiss des Europaparlaments stellt zwar stellenweise eine deutliche Verbesserung gegenüber dem Entwurf der Europäischen Kommission dar. Insbesondere mit den erstmals verankerten Anreizelementen für pseudonyme Datenverarbeitung wird europaweit ein Modell eingeführt, das in Deutschland bereits seit langem erfolgreich eingesetzt wird. Diese Regelungen sind vor allem für kleine Diensteanbieter im Wettbewerb überlebenswichtig. Jedoch fehlte dem Parlament der Mut, diesen „privacy by design“-Ansatz im Gesetzestext in aller Deutlichkeit zu regeln. Stattdessen wurde er in so genannten Erwägungsgründen versteckt und damit Rechtsunsicherheit provoziert.

Als kontraproduktiv bewertet der BVDW die Regelung zum so genannten Profiling. Der Kompromiss führt in der jetzigen Fassung dazu, dass sich das geplante Verbot auch auf Daten erstreckt, die keinen Personenbezug haben. Dies stellt weder eine angemessene, abgestufte Lösung dar, die das tatsächliche Risiko und die Sensibilität der betroffenen Daten berücksichtigt, noch ist dieser Vorschlag geeignet, in der Praxis für mehr Datenschutz zu sorgen.

\section{Ausschreibung des 5. Deutschen IT-Sicherheitspreises der Horst Görtz-Stiftung}

Die Horst Görtz Stiftung schreibt zum fünften Mal den mit insgesamt 200.000 Euro dotierten Deutschen IT-Sicherheitspreis aus. Die Schirmherrschaft hat Michael Hange, Präsident des Bundesamtes für Sicherheit in der Informationstechnik, übernommen.

Mit dem Deutschen IT-Sicherheitspreis soll IT-Sicherheit "Made in Germany" gefestigt und die Innovationskraft der deutschen Wirtschaft gestärkt werden. Die Durchführung des fünften Deutschen IT-Sicherheitspreises wird unterstützt durch das Horst Görtz Institut für IT-Sicherheit der Ruhr-Universität Bochum.

Teilnahmeberechtigt sind Forscher und Entwickler sowie Forschungs- und Entwicklungs-Teams in Universitäten, Forschungsinstituten und Unternehmen. Auswahlkriterien sind sowohl Originalität als auch wirtschaftliche Umsetzbarkeit.

Bis zum 15. Februar 2014 können Interessenten ihre Skizzen einreichen. 